\title{
Intestinal parasites co-infection and associated factors among active pulmonary tuberculosis patients in selected health centers, Addis Ababa, Ethiopia: unmatched case control study
}

\author{
Ayinalem Alemu ${ }^{1,2^{*}}$, Abebaw Kebede 1 , Biniyam Dagne ${ }^{1}$, Misikir Amare ${ }^{1}$, Getu Diriba', Bazezew Yenew ${ }^{1}$,
} Ephrem Tesfaye ${ }^{1}$, Mengistu Tadesse ${ }^{1}$, Waganeh Sinshaw ${ }^{1}$, Dawit Challa ${ }^{1}$ and Kassu Desta ${ }^{2}$

\begin{abstract}
Background: In co-endemic areas, rate of intestinal parasites and tuberculosis (TB) co-infection thought to be high. However, there are limited studies on the epidemiology of this co-infection in Ethiopia. Therefore, the present study aimed to generate evidence on intestinal parasites co-infection rate and associated factors among pulmonary tuberculosis patients (PTB) and their household contacts in Addis Ababa, Ethiopia.

Methods: Unmatched case-control study was conducted. Data were collected from 91 PTB patients (cases) and 89 household contacts (controls). Socio-demographic characteristics and associated factors were collected using structured questionnaire. Sputum, stool and blood specimens were collected, processed and examined for PTB, intestinal parasites and Human Immunodeficiency virus anti-body test, respectively. Data were entered and analyzed by Statistical Packages for Social Sciences (SPSS) Version 20. Descriptive statistics, Fisher's exact test, binary logistic regression, and odds ratio were used. P-value of $<0.05$ was considered as statistically significant.

Results: The infection rate of intestinal parasites based on one stool samples in PTB patients and controls was 22 and $9 \%$, respectively. The difference was statistically significant ( $C O R=2.85 ; 95 \% \mathrm{Cl}=1.18-6.87$ ). The most prevalent intestinal parasite in PTB patients was Gardia lamblia $(8.8 \%, 8)$, followed equally by Ascaris lumbricoides, Haymenolopsis nana and Entamoeba histolytica/dispar $(4.4 \%$, 4). Co-infection in PTB patients was associated with body mass index (BMI) $<18.5$ (AOR $=6.71 ; 95 \% \mathrm{Cl}=1.65-27.25)$ and dirty material in finger nails (AOR $=8.99 ; 95 \% \mathrm{Cl}=2.46-32.78)$. There was no variable associated with parasitic infections in controls in our analysis, which might be due to the low prevalence of intestinal parasites'.
\end{abstract}

Conclusions: There was a statistical significant difference in the infection rate of intestinal parasites in PTB patients compared to healthy household contacts. The consequence of co-infection on developing an active disease, disease severity and treatment efficacy needs to be investigated in future.

Keywords: Pulmonary tuberculosis, Intestinal parasites, Co-infection, Associated factors

\footnotetext{
* Correspondence: ayinalemal@gmail.com

${ }^{1}$ Ethiopian Public Health Institute, Addis Ababa, Ethiopia

${ }^{2}$ Department of Medical Laboratory Science, College of Health Sciences,

Addis Ababa University, Addis Ababa, Ethiopia
}

(c) The Author(s). 2019 Open Access This article is distributed under the terms of the Creative Commons Attribution 4.0 International License (http://creativecommons.org/licenses/by/4.0/), which permits unrestricted use, distribution, and reproduction in any medium, provided you give appropriate credit to the original author(s) and the source, provide a link to the Creative Commons license, and indicate if changes were made. The Creative Commons Public Domain Dedication waiver (http://creativecommons.org/publicdomain/zero/1.0/) applies to the data made available in this article, unless otherwise stated. 


\section{Background}

Tuberculosis is the leading cause of death from a single infectious agent globally [1]. The problem is worse in high burden countries [1]. The 30 high TB, multi drug resistance-TB and TB/ Human Immunodeficiency virus (HIV) burden Countries accounted $90 \%$ of all estimated incident cases worldwide [1]. Although one third of the world population is estimated to be infected with Mycobacterium tuberculosis (MTB), relatively a small proportion develops TB [1-3]. The host's immune system is a key factor for the progression of MTB infection [4, 5]. Susceptibility to the disease is associated with reduced Th1 type responses [3]. It was reported that intestinal parasites (IP), specifically helminthes causes immune activation with biased $\mathrm{T}$ helper 2 responses and down regulated Th1 and Cytolytic $\mathrm{T}$ lymphocytes activity [6]. This change in the immunological milieu of the host might impair the immunological response to pathogens which need Th1 responses to limit the severity and progression of infection [6]. In sub-Saharan Africa, where the prevalence of parasitic infections is very high, a dominant Th2 polarized immune response has been reported and suggested to increase susceptibility to $M T B[3,7]$.

It is estimated that nearly 3.5 billion people are affected, and 450 million are ill due to parasite (protozoa or helminthes) infections globally [5, 8]. TB and IPs affect primarily low social and economic level populations [5]. Both infections overlap substantially in geographic distribution $[3,9]$. Thus, the likely hood of co-infection is thought to be high. Epidemiology of this co-infection was reported in different countries $[3,5,7,9,11-15]$. Both infections are the major public health problems at national and regional level in Ethiopia [6, 11]. Higher prevalence of IPs co-infection among TB patients was reported from Gondar, Ethiopia [3]. In a recent study from Arbaminch, Ethiopia a $26.3 \%$ of intestinal helminthes co-infection rate was reported [12]. This co-infection would increase the complexity of control and prevention of TB and parasitic diseases [9-16]. It is described that Bacille Calmette Guerin (BCG) has a limited effect against TB epidemic in developing countries where the prevalence of intestinal helmenthiasis is high [16-18].

Understanding co-infection rate has an input to design effective mechanism to reduce mortality due to dual effect and for designing effective prevention and control mechanisms. However, there are limited studies in the country which are concentrated in similar settings. Therefore, the present study aimed to assess intestinal parasite co-infection rate and associated factors among active PTB patients in highly populous Sub-city of Addis Ababa (urban setting), Ethiopia.

\section{Methods}

\section{Study setting and design}

Unmatched case-control study was conducted during the period between Jan 2017 to Jan 2018, in selected health centers of Kolfe Keraniyo Sub-city, Addis Ababa, Ethiopia. Addis Ababa is the capital city of Ethiopia, with an altitude ranging from $2100 \mathrm{~m}$ to $3000 \mathrm{~m}$ above sea level [19]. Among the ten Sub-cities in the city, Kolfe Keraniyo Sub-city was selected purposely due to easier access to health centers and patient flow. Three health centers which provide care and treatment for TB and HIV/AIDS patients were randomly selected, namely; Kolfe, Wereda 11 and Lomi meda health centers.

\section{Eligibility criteria and sampling}

All PTB patients visiting TB clinics and their household contacts were used as study subjects. Cases were individuals with bacteriological confirmed active PTB such that all individuals with smear positive results were included. For confirmation Culture and Xpert MTB/RIF Assay were performed for all cases and included after confirmation. Controls were healthy household contacts to active PTB patients with no clinically and bacteriological diagnosed TB. All age groups who fullfilled the definition of cases/controls, volunteered to take part and gave written informed consent were included in the study. However, HIV positive individuals and TB patients on treatment excluded from the study. All controls were screened for TB symptoms and PTB was ruled out by using a combination of bacteriological diagnostic methods; Smear microscopy, Culture and Xpert MTB/RIF Assay. Sample size of 178 ( 89 cases, 89 controls) was obtained by using OpenEpi, version 3 sample size calculation for unmatched case control study by Fleiss with continuity correction (prevalence in cases; 50, prevalence in controls; $28.5, \alpha ; 0.05$, power; 80 , ratio; $1: 1$ ), based on an average of two studies conducted at Gondar, Ethiopia [3, 7]. Nonrandom convenience sampling technique was used; such that consecutive cases and controls were enrolled. Written informed consent was administered for volunteers after full explanation of the study. Socio-demographic characteristics and associated factors were collected using a pretested structured questionnaire via face to face interview by trained nurses. BCG vaccination status was collected by nurses by looking BCG scar on participants' arms. Shoe wearing was defined as a habit of frequent shoe wearing any where being outside home. Similarly presence of any foreign body in the participant's finger nail (untrimmed) was considered as presence of dirty material. Laboratory examinations including sputum smear microscopy, Xpert MTB/RIF assay, sputum culture, stool examination and HIV antibody test were done by senior laboratory technologists.

\section{Laboratory diagnosis}

Two spot sputum specimens for smear microscopy and one morning sputum specimen volume of $5 \mathrm{ml}$ for Xpert MTB/RIF assay and culture were collected based on 
Ethiopian TB and leprosy control guideline [20]. Smear microscopy was done by using auramine $\mathrm{O}$ staining method and examined by fluorescent microscope [21]. Sputum samples for culture and Xpert MTB/RIF assay were stored at $2-8^{\circ} \mathrm{c}$ until transported to Ethiopian Public Health Institute, National Tuberculosis Reference Laboratory. Samples were processed by N-Acetyl-L-Cysteine-Sodium Hydroxide (NALC-NaOH) decontamination method as described by GLI mycobacteriology laboratory manual [22]. Phosphate buffere ( $\mathrm{pH}$ 6.8) solution (PBS) was used to for homogenization and re suspension. Re-suspended sediment was inoculated in to both solid (LJ) and liquid (MGIT) medias. Prior to inoculation, $800 \mu \mathrm{l}$ from a mix of $15 \mathrm{ml}$ MGIT growth supplement and PANTA was added to MGIT tubes [22]. LJ slants were incubated at $37^{\circ} \mathrm{C}$, while MGIT tubes were loaded to MGIT 960 instrument (Becton-Dickinson and Company, Sparks MD). LJ slants not had colonies growing at eighth weeks and no growth unit on MGIT tubes at 42 days were defined as negative [22, 23]. SD BIOLINE TB Ag MPT 64\%(STANDARD DIAGNOSTICS, INC, Republic of Korea) test was used for the confirmation of Mycobacterium tuberculosis complex [24]. For Xpert MTB/RIF assay $0.5 \mathrm{ml}$ of sediment was mixed with $1.5 \mathrm{ml}$ of sample reagent buffer (supplied in kit). After $15 \mathrm{~min}$, an approximate of $2 \mathrm{ml}$ of the specimen was dispensed it into Xpert MTB/RIF's cartridge and loaded to GeneXpert instrument (Cepheid, Sunnyvale, CA, USA). The result was viewed after $2 \mathrm{~h}$ [25].
An approximate of a three gram of stool sample was collected and processed for parasitic examination using Wet mount, Formol-ether concentration and Modified $\mathrm{ZN}$ method. For wet mount; an approximate of $50 \mathrm{mg}$ of feces mixed with a drop of normal saline on a clean slide and screened systematically for the presence of helminth ova and larvae or protozoan cysts and trophozoites [26]. For formol-ether concentration, about $1 \mathrm{~g}$ of faeces was placed in a clean $15 \mathrm{ml}$ conical centrifuge tube containing $7 \mathrm{ml}$ formalin saline. The suspension was filtered through a sieve into another conical centrifuge tube and $3 \mathrm{ml}$ of diethyl ether was added. The contents were centrifuged at 3, $200 \mathrm{rpm}$ for $3 \mathrm{~min}$ and the supernatant was poured away. Two smears were made on clean glass slides and the entire area of one smear was systematically examined by using $10 \mathrm{x}$ and $40 \mathrm{x}$ objective lenses [11]. From the other smear, modified $\mathrm{ZN}$ staining method was used to stain coccidians. After fixing the smear with methanol; $1 \%$ carbol fuchsin, $1 \% \mathrm{v} / \mathrm{v}$ acid alcohol and $0.25 \%$ malachite green were used as a primary stain, decolorizer and counter stain respectively. The smear was examined by experienced microbiologist using 100x objective [27].

HIV antibody test on TB patients was done by using rapid test kits (Wanti HIV1 + 2, Uni-Gold ${ }^{\mathrm{mi}} \mathrm{HIV}$ and VIKIA HIV 1/2 [28]. Interpretation was based on the combination of these three rapid tests such as Wanti HIV1 +2 as a screening test, Uni-Gold ${ }^{\mathrm{m}}$ HIV as a

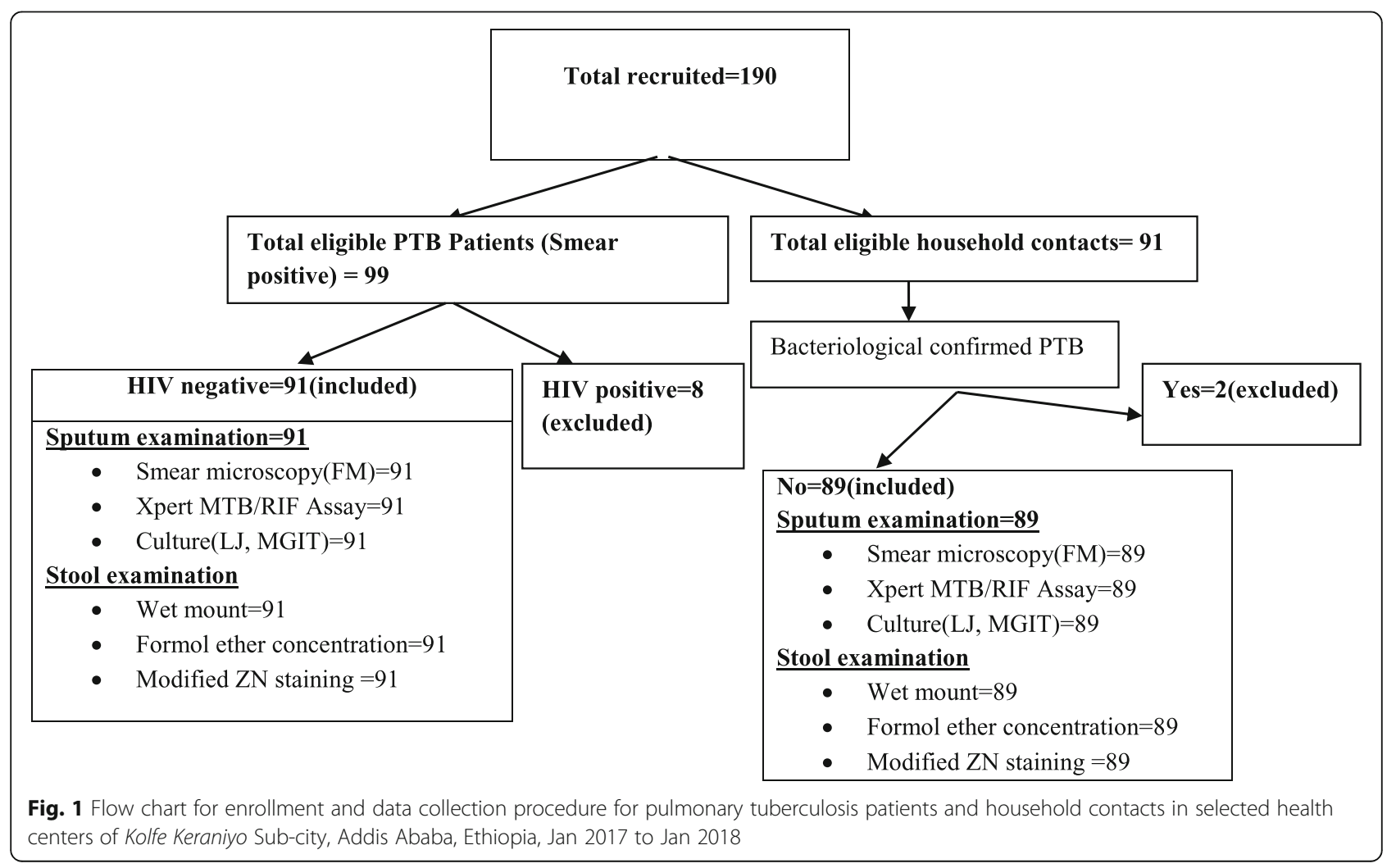


Table 1 Socio-demographic and behavioral characteristics of tuberculosis patients $(n=91)$ and controls $(n=89)$ in selected health centers of Kolfe Keraniyo Sub-city, Addis Ababa, Ethiopia, Jan 2017 to Jan 2018

\begin{tabular}{|c|c|c|c|}
\hline & Variables & $\begin{array}{l}\text { Cases } \\
\text { Number (\%) }\end{array}$ & $\begin{array}{l}\text { Controls } \\
\text { Number (\%) }\end{array}$ \\
\hline \multirow[t]{2}{*}{ Age } & Mean & 26.7 & 26.96 \\
\hline & SD & 7.69 & 9.19 \\
\hline \multirow[t]{4}{*}{ Age group } & $\leq 24$ & $44(48.4)$ & $38(42.7)$ \\
\hline & $25-34$ & $31(34.1)$ & $34(38.2)$ \\
\hline & $\geq 35$ & 16(17.6) & $17(19.1)$ \\
\hline & Total & $91(100.0)$ & $89(100.0)$ \\
\hline \multirow[t]{3}{*}{ Sex } & Male & $56(61.5)$ & $33(37.1)$ \\
\hline & Female & $35(38.5)$ & $56(62.9)$ \\
\hline & Total & $91(100.0)$ & $89(100.0)$ \\
\hline \multirow[t]{3}{*}{ Residence } & Urban & 89(97.8) & $87(97.8)$ \\
\hline & Rural & $2(2.2)$ & $2(2.2)$ \\
\hline & Total & $91(100.0)$ & $89(100.0)$ \\
\hline \multirow[t]{3}{*}{ Marital status } & Single & $56(61.5)$ & $43(48.3)$ \\
\hline & Married & $35(38.5)$ & $46(51.7)$ \\
\hline & Total & $91(100.00)$ & $89(100.0)$ \\
\hline \multirow[t]{5}{*}{ Educational status } & No formal education & $26(28.6)$ & $27(30.3)$ \\
\hline & Primary completed & $42(46.2)$ & $25(28.1)$ \\
\hline & $\begin{array}{l}\text { High school } \\
\text { completed }\end{array}$ & 19(20.9) & $20(22.5)$ \\
\hline & College \&above & $4(4.4)$ & 17(19.1) \\
\hline & Total & $91(100.0)$ & $89(100.0)$ \\
\hline \multirow[t]{4}{*}{ Monthly income } & Low & $46(50.5)$ & 28(31.5) \\
\hline & Medium & $22(24.2)$ & $25(28.1)$ \\
\hline & Satisfactory & $23(25.3)$ & $36(40.5)$ \\
\hline & Total & $91(100.0)$ & $89(100.0)$ \\
\hline \multirow[t]{3}{*}{ Latrine availability } & Yes & 85(93.4) & $89(100.0)$ \\
\hline & No & $6(6.6)$ & $0(0.0)$ \\
\hline & Total & $91(100.0)$ & $89(100.0)$ \\
\hline \multirow[t]{3}{*}{ Raised poultry } & Yes & $8(8.8)$ & $22(24.7)$ \\
\hline & No & 83(91.2) & $67(75.3)$ \\
\hline & Total & $91(100.0)$ & $89(100.0)$ \\
\hline \multirow[t]{3}{*}{ Occupation } & Employed & $31(34.1)$ & $30(33.7)$ \\
\hline & Unemployed & $60(65.9)$ & $59(66.3)$ \\
\hline & Total & $91(100.0)$ & $89(100.0)$ \\
\hline \multirow[t]{3}{*}{ Swimming habit } & Yes & $6(6.6)$ & $3(3.4)$ \\
\hline & No & $85(93.4)$ & 86(96.6) \\
\hline & Total & $91(100.0)$ & $89(100.0)$ \\
\hline \multirow[t]{3}{*}{ Habit of shoe wearing } & Yes & $91(100.0)$ & 88(98.9) \\
\hline & No & $\mathrm{O}(0,0)$ & $1(1.1)$ \\
\hline & Total & $91(100.0)$ & $89(100.0)$ \\
\hline
\end{tabular}

Table 1 Socio-demographic and behavioral characteristics of tuberculosis patients $(n=91)$ and controls $(n=89)$ in selected health centers of Kolfe Keraniyo Sub-city, Addis Ababa, Ethiopia, Jan 2017 to Jan 2018 (Continued)

\begin{tabular}{|c|c|c|c|}
\hline & Variables & $\begin{array}{l}\text { Cases } \\
\text { Number (\%) }\end{array}$ & $\begin{array}{l}\text { Controls } \\
\text { Number (\%) }\end{array}$ \\
\hline \multirow[t]{4}{*}{ Bathing } & Home & 87(95.6) & $87(97.8)$ \\
\hline & River & $4(4.4)$ & $1(1.1)$ \\
\hline & Home and River & $0(0.0)$ & $1(1.1)$ \\
\hline & Total & $91(100.0)$ & $89(100.0)$ \\
\hline \multirow{3}{*}{$\begin{array}{l}\text { Hand wash before } \\
\text { meal }\end{array}$} & Yes & $91(100.0)$ & $89(100.0)$ \\
\hline & No & $0(0.0)$ & $0(0.0)$ \\
\hline & Total & $91(100.0)$ & $89(100.0)$ \\
\hline \multirow[t]{4}{*}{ Water source for drink } & Tap & 89(97.8) & 87(97.8) \\
\hline & River & $\mathrm{O}(0.0)$ & $0(0.0)$ \\
\hline & Tap and River & $2(2.2)$ & $2(2.2)$ \\
\hline & Total & $91(100.0)$ & $89(100.0)$ \\
\hline \multirow[t]{4}{*}{ Washing cloth } & Home & 85(93.4) & 88(98.9) \\
\hline & River & $2(2.2)$ & $0(0.0)$ \\
\hline & Home and River & $4(4.4)$ & $1(1.1)$ \\
\hline & Total & $91(100.0)$ & $89(100.0)$ \\
\hline \multirow{3}{*}{$\begin{array}{l}\text { Dirty material in } \\
\text { the finger }\end{array}$} & Yes & $21(23.1)$ & 13(14.6) \\
\hline & No & 70(76.9) & $76(85.4)$ \\
\hline & Total & $91(100.0)$ & $89(100.0)$ \\
\hline \multirow{3}{*}{$\begin{array}{l}\text { Eating unwashed } \\
\text { vegetables }\end{array}$} & Yes & $28(30.8)$ & 19(21.3) \\
\hline & No & $63(69.2)$ & 70(78.7) \\
\hline & Total & $91(100.0)$ & $89(100.0)$ \\
\hline \multirow[t]{3}{*}{ Eating raw meat } & Yes & $52(57.1)$ & $38(42.7)$ \\
\hline & No & $39(42.9)$ & $51(57.3)$ \\
\hline & Total & $91(100.0)$ & $89(100.0)$ \\
\hline
\end{tabular}

confirmatory test and Uni-Gold ${ }^{\mathrm{mi}}$ HIV and VIKIA HIV $1 / 2$ was used as a tie breaker.

\section{Data quality control}

A structured questionnaire was prepared and pre-tested on patients who did not include in the study. Nurses working in TB clinics and laboratory technologists of each health center were selected and trained. The collected data were checked for completeness, accuracy, clarity,and consistency by the principal investigator, on daily basis. Controls were run and preventive maintenance was performed to ensure the quality of instrument performance. Reagents were checked for reliability and reproducibility of the test before any test started. Appropriate sputum and stool specimens were collected, processed and examined according to standard operating procedures. For all smear positive PTB patients, Xpert 
MTB/RIF and sputum culture were performed for confirmation. Stool examination was conducted by individuals who were blinded to the source of the specimen whether it was from TB patients or from controls. Control slides were used. The result of laboratory examination was recorded on well-prepared format carefully. Prepared LJ media was checked visually for the color, texture,and homogeneity, and performance check and sterility test were performed before usage. For Liquid culture, everything was done based on MGIT instrument manual [29].

\section{Statistical analysis}

Data were coded, checked, entered, stored andanalyzed by SPSS Ver. 20. Q-Q plot was used to check normal distribution for age, BMI and monthly income. Descriptive statistics such as frequencies, proportions, mean and standard deviation were used to explain socio-demographic, associated factors and intestinal parasites infection rate. The association between intestinal parasites and TB co-infection was evaluated by using binary logistic regression. To analyze associated factors for the co-infection univariate analysis (binary logistic regression) was done. For all variables with a $P$-value of $\leq 0.28$ in the univariate analysis, multivarate analysis was done sequentially by removing the variable with largest $P$-value until statistically significant associations were found. Fisher's exact test was used to compare the study variables for the presence or absence of association for variables not fulfilled the assumptions of Chi-squared test. Odds ratio and 95\% confidence interval was used to measure the strength of an association. $P$-value $<0.05$ was considered indicative of a statistically significant difference.

\section{Results}

\section{Socio-demographic characteristics}

A total of 190 study participants were enrolled in this study; of them 99 were cases with bacteriological confirmed active PTB and 91 were controls that were healthy household contacts to TB patients. Eight from the cases and two from the controls were excluded due to HIV co-infection and bacteriologically confirmed PTB respectively. Therefore, 91 cases and 89 controls were included in the analysis (Fig. 1). Majority (61.5\%) among the cases were males, while females accounted largest proportions $(62.9 \%)$ among the controls. The mean age of cases and controls was $26.7( \pm 7.7)$ and $26.69( \pm 9.19)$ years, respectively. More than $90 \%$ of cases and controls were younger than 40 years. The residence for $97.8 \%$ of both cases and controls were from urban. Around 66\% from the cases and the controls were unemployed. Both cases and controls had been utilizing tap water as a source of drinking water equally $(97.8 \%)$. Eighty five
(93.4\%) from cases and all of the controls reported that they had latrine or use latrine. All cases and majority of the controls $(98.9 \%)$ had a shoe wearing habit. Similarly all from both groups have been washing their hands before taking meal, at breakfast, lunch and dinner time. A habit of hand washing practice after toilet was reported by $82.4 \%$ of cases and $85 \%$ of controls. However, $40 \%$ of cases and $41.2 \%$ of controls were washing their hands by using water without any soap or detergent. In $21(23.1 \%)$ cases and $13(14.6 \%)$ controls there was a dirty material in their finger nails (Table 1). The mean body Mass Index (BMI) of PTB patients was $18.26( \pm 2.14)$. Among PTB patients $17.6 \%$ had a scar on their arm for BCG vaccination. Among house hold contacts, 19.1\% had a scar.

Table 2 Infection rate of intestinal parasites among tuberculosis patients $(n=91)$ and controls $(n=89)$ in selected health centers of Kolfe Keraniyo Sub-city, Addis Ababa, Ethiopia, Jan 2017 to Jan 2018

\begin{tabular}{|c|c|c|c|}
\hline Variables & $\begin{array}{l}\text { Cases } \\
\text { Number (\%) }\end{array}$ & $\begin{array}{l}\text { Controls } \\
\text { Number (\%) }\end{array}$ & $\operatorname{COR}(95 \% \mathrm{Cl})$ \\
\hline \multicolumn{4}{|l|}{ Parasites } \\
\hline No & $71(78.0)$ & $81(91.0)$ & 1.00 \\
\hline Yes & $20(22.0)$ & $8(9.0)$ & $2.85(1.18-6.87)$ \\
\hline Total & $91(100.0)$ & $89(100.0)$ & \\
\hline Protozoa & & & 1.00 \\
\hline No & 79(86.8) & 83(93.3) & $2.10(0.75-5.87)$ \\
\hline Yes & $12(13.2)$ & $6(6.7)$ & \\
\hline Total & $91(100.0)$ & $89(100.0)$ & \\
\hline \multicolumn{4}{|l|}{ Helminthes } \\
\hline No & $81(89.0)$ & $87(97.75)$ & 1.00 \\
\hline Yes & 10(11.0) & $2(2.25)$ & $5.37(1.14-25.25)$ \\
\hline Total & $91(100.0)$ & $89(100.0)$ & \\
\hline$\geq 2$ parasites & & & - \\
\hline No & 87(95.6) & $89(100.0)$ & \\
\hline Yes & $4(4.4)$ & $0(0.0)$ & \\
\hline Total & $91(100.0)$ & $89(100.0)$ & \\
\hline \multicolumn{4}{|l|}{ Each parasite } \\
\hline E.histolytca/dispar & $4(16.7)$ & $5(62.5)$ & \\
\hline G.lamblia & $8(33.3)$ & $1(12.5)$ & $8.48(1.04-69.29)$ \\
\hline C. parvum & $2(8.3)$ & $0(0.0)$ & \\
\hline A.lumbricoids & $4(16.7)$ & $0(0.0)$ & - \\
\hline S.sterocolaris & $0(0.0)$ & $1(12.5)$ & \\
\hline Taenia spp & $0(0.0)$ & $1(12.5)$ & \\
\hline H.nana & $4(16.7)$ & $0(0.0)$ & - \\
\hline T.trichuria & $2(8.3)$ & $0(0.0)$ & \\
\hline Total & $24(100.0)$ & $8(100.0)$ & \\
\hline
\end{tabular}

COR Crude Odds ratio, Cl Confidence Interval," -" = Not Done 
The burden of intestinal parasites

The overall infection rate of intestinal parasites among PTB patients and controls was 22\%(20/91) and 9\% (8/89) respectively. The difference was statistically significant (COR
$=2.85 ; 95 \% \mathrm{CI}=1.18-6.87) . \mathrm{A}$ total of 24 intestinal parasites (14 intestinal protozoans and 10 intestinal helminthes) from PTB patients and eight intestinal parasites (six intestinal protozoans and two intestinal helminthes) from

Table 3 Associated factors for intestinal parasites co-infection among tuberculosis patients $(n=91)$ in selected health centers of Kolfe Keraniyo Sub-city, Addis Ababa, Ethiopia, Jan 2017 to Jan 2018

\begin{tabular}{|c|c|c|c|c|c|c|}
\hline \multirow[t]{3}{*}{ Variables } & \multicolumn{6}{|l|}{ Cases } \\
\hline & \multicolumn{2}{|l|}{ Stool examination result } & \multirow[t]{2}{*}{$\operatorname{COR}(95 \% \mathrm{Cl})$} & \multirow[t]{2}{*}{$p$-value } & \multirow[t]{2}{*}{$\mathrm{AOR}(95 \% \mathrm{Cl})$} & \multirow[t]{2}{*}{$p$-value } \\
\hline & Parasites detected, $\mathrm{N}^{*}(\%)$ & Parasites not detected, $\mathrm{N}^{*}(\%)$ & & & & \\
\hline Gender & & & & 0.873 & - & - \\
\hline Male & $12(60.0)$ & $44(62.0)$ & 1.00 & & & \\
\hline Female & $8(40.0)$ & 27(38.0) & $1.08(0.39-2.99)$ & & & \\
\hline Age group & & & & 0.700 & - & - \\
\hline $10-24$ & $8(40.0)$ & $36(50.7)$ & $0.66(0.17-2.16)$ & & & \\
\hline $25-34$ & $8(40.0)$ & 23(32.4) & $1.04(0.26-4.18)$ & & & \\
\hline$\geq 35$ & $4(20.0)$ & 12(16.9) & 1.00 & & & \\
\hline BMl & & & & 0.025 & & 0.008 \\
\hline$<18.49$ & 16(80.0) & $36(50.7)$ & $3.88(1.18-12.78)$ & & $6.71(1.65-27.25)$ & \\
\hline $18.5-24.99$ & $4(20.0)$ & $35(49.3)$ & 1.00 & & 1.00 & \\
\hline Marital status & & & & 0.381 & - & \\
\hline Single & 14(70.0) & $42(59.2)$ & 1.00 & & & \\
\hline Married & $6(30.0)$ & $29(40.8)$ & $0.62(0.21-1.80)$ & & & \\
\hline Educational status & & & & 0.329 & - & - \\
\hline No formal education & $4(20.0)$ & $22(31.0)$ & 1.00 & & & \\
\hline Primary completed & $8(40.0)$ & $34(47.9)$ & $1.29(0.34-4.81)$ & & & \\
\hline Completed high school & $6(30.0)$ & 13(18.3) & $2.53(0.60-10.70)$ & & & \\
\hline Collage \& above & $2(10.0)$ & $2(2.8)$ & $5.50(0.59-51.19)$ & & & \\
\hline Occupation & & & & 0.141 & & 0.366 \\
\hline Employed & $4(20.0)$ & $27(38.0)$ & 1.00 & & 1.00 & \\
\hline Unemployed & 16(80.0) & $44(62.0)$ & $2.45(0.74-8.11)$ & & $1.86(0.48-7.22)$ & \\
\hline Monthly income & & & & 0.280 & & 0.186 \\
\hline Low & $12(60.0)$ & $34(47.9)$ & $1.00(0.32-3.12)$ & & $1.46(0.34-6.14)$ & \\
\hline Medium & $2(10.0)$ & $20(28.1)$ & $0.28(0.05-1.59)$ & & $0.23(0.03-1.73)$ & \\
\hline Satisfactory & $6(30.0)$ & $17(24.0)$ & 1.00 & & & \\
\hline Dirty material in the finger & & & & 0.002 & & 0.001 \\
\hline Yes & $10(50.0)$ & $11(15.5)$ & $5.45(1.83-16.17)$ & & $8.99(2.46-32.78)$ & \\
\hline No & $10(50.0)$ & $60(84.5)$ & 1.00 & & 1.00 & \\
\hline Eating unwashed vegetables & & & & 0.933 & - & \\
\hline Yes & 14(70.0) & $22(31.0)$ & $0.95(0.32-2.81)$ & & & \\
\hline No & $6(30.0)$ & $49(69.0)$ & 1.00 & & & \\
\hline Eating raw meet & & & & 0.466 & - & - \\
\hline Yes & $10(50.0)$ & $22(31.0)$ & $0.69(0.25-1.87)$ & & & \\
\hline No & 10(50.0) & $49(69.0)$ & 1.00 & & & \\
\hline Raised poultry/livestock & & & & 0.829 & - & - \\
\hline Yes & $2(10.0)$ & $6(8.5)$ & $1.20(0.22-6.48)$ & & & \\
\hline No & 18(90.0) & 65(91.5) & 1.00 & & & \\
\hline
\end{tabular}


controls were identified. There was a statistical significance difference for helminthic infection between PTB patients and controls $(\mathrm{COR}=5.37 ; 95 \% \mathrm{CI}=1.14,25.25)$, but not for protozoans $(p=0.157)$. Multiple parasitic infections were found in four (4.4\%) PTB patients; but not in controls $(p=$ 0.045). Among all intestinal parasites identified in PTB patients; G.lamblia was frequently found $(8,33.3 \%)$, followed by the equal prevalence of $A$. lumbricoides, H.nana and E.histolytica/dispar (4, 16.7\%). E.histolytica/dispar was frequently identified from controls $(5,62.5 \%)$. Among all intestinal parasites identified G.lamblia, A.lumbricoides and H.nana were found with a statistical significance difference ( $p=0.046, p=0.045$ and $p=0.045$ respectively) between cases and controls (Table 2).

\section{Associated factors for intestinal parasites infection}

Intestinal parasitic co-infection among PTB patients had a statistically significant association BMI (AOR = $6.71 ; 95 \% \mathrm{CI}=1.65-27.25)$ and presence of dirty material in the participant's finger $(\mathrm{AOR}=8.99 ; 95 \% \mathrm{CI}=2.46-$ 32.78) (Table 3). However; Intestinal parasitic infection in the control group had not statistically significant association with any of socio-demographic or behavioral factors (Table 4, Table 5). Intestinal parasites are identified from 6(37.5\%) of BCG vaccinated PTB patients and the majority $(4,66.7 \%)$ were intestinal helminthes.

\section{Discussion}

TB and parasitic diseases overlap similar geographic distribution, especially in developing countries $[7,16]$. The overall intestinal parasite co-infection rate among active PTB patients in this study was $22 \%$. This is similar with studies done at Arbaminch, Ethiopia [12] and Brazil [5]. However, it is lower than other studies in Gondar, Ethiopia [3, 13] and Brazil [14]. Three consecutive stool specimens examined in these studies might be a reason for the lower results in the present study. These studies were conducted 15 years ago which might be another possible reason, because currently health extension workers are engaged in the primary health care activities across the nation. Inaddition, co-infection was lower than other studies conducted in Gondar, Ethiopia [7, 11]

Table 4 Associated factors for intestinal parasites co-infection among healthy household contacts $(n=89)$ in selected health centers of Kolfe Keraniyo Sub-city, Addis Ababa, Ethiopia, Jan 2017 to Jan 2018

\begin{tabular}{|c|c|c|c|c|}
\hline \multirow[t]{3}{*}{ Variables } & \multicolumn{4}{|l|}{ Controls } \\
\hline & \multicolumn{2}{|l|}{ Stool examination result } & \multirow[t]{2}{*}{$\operatorname{COR}(95 \% \mathrm{Cl})$} & \multirow[t]{2}{*}{$p$-value } \\
\hline & Parasites detected, $\mathrm{N}^{*}(\%)$ & Parasites not detected, $\mathrm{N}^{*}(\%)$ & & \\
\hline Gender & & & & 0.979 \\
\hline Male & $3(37.5)$ & $30(37.0)$ & $1.02(0.22-4.57)$ & \\
\hline Female & $5(62.5)$ & $51(63.0)$ & 1.00 & \\
\hline Educational status & & & & 0.387 \\
\hline $1^{\circ}$ completed or less & $4(50.0)$ & 48(59.2) & $0.38(0.07-1.94)$ & \\
\hline Completed high school & $1(12.5)$ & $19(23.5)$ & $0.24(0.02-2.61)$ & \\
\hline College $\&$ above & $3(37.5)$ & 14(17.3) & 1.00 & \\
\hline Occupation & & & & 0.315 \\
\hline Employed & $4(50.0)$ & $26(32.1)$ & 1.00 & \\
\hline Unemployed & $4(50.0)$ & $55(67.9)$ & $0.47(0.11-2.04)$ & \\
\hline Dirty material in the finger & & & & 0.392 \\
\hline Yes & $2(25.0)$ & $11(15.6)$ & $2.12(0.37-11.86)$ & \\
\hline No & $6(75.0)$ & $70(86.4)$ & 1.00 & \\
\hline Eating unwashed vegetables & & & & 0.254 \\
\hline Yes & $3(37.5)$ & 16(19.8) & $2.43(0.52-11.28)$ & \\
\hline No & $5(62.5)$ & $65(80.2)$ & 1.00 & \\
\hline Eating raw meat & & & & 0.247 \\
\hline Yes & $5(62.5)$ & $33(40.7)$ & $2.42(0.54-10.84)$ & \\
\hline No & $3(37.5)$ & $48(59.3)$ & 1.00 & \\
\hline Raised poultry/livestock & & & & 0.415 \\
\hline Yes & $1(12.5)$ & 21(25.9) & $0.40(0.04-3.51)$ & \\
\hline No & $7(87.5)$ & $60(74.1)$ & 1.00 & \\
\hline
\end{tabular}

COR Crudes Odds Ratio, Cl Confidence Interval, $\mathrm{N}^{*}(\%)=$ Number 
Table 5 Associated factors for intestinal parasites co-infection among tuberculosis patients $(n=91)$ and controls $(n=89)$ in selected health centers of Kolfe Keraniyo Sub-city, Addis Ababa, Ethiopia, Jan 2017 to Jan 2018

\begin{tabular}{|c|c|c|c|c|c|c|}
\hline \multirow[t]{2}{*}{ Variables } & \multicolumn{3}{|l|}{ Cases } & \multicolumn{3}{|l|}{ Controls } \\
\hline & Number (\%) & Fisher's exact test & $p$-value & Number (\%) & Fisher's exact test & $p$-value \\
\hline Residence & & 7.26 & 0.046 & & 0.00 & 1.000 \\
\hline Urban & 18(90.0) & & & $8(100.0)$ & & \\
\hline Rural & $2(10.0)$ & & & $0(100.0)$ & & \\
\hline Latrine availability & & 0.69 & 0.44 & & - & - \\
\hline Yes & $20(100.0)$ & & & $8(100.0)$ & & \\
\hline No & $0(0.0)$ & & & $0(0.0)$ & & \\
\hline Swimming habit & & 0.69 & 0.404 & & 0.22 & 0.636 \\
\hline Yes & $0(0.0)$ & & & $1(12.5)$ & & \\
\hline No & $20(100.0)$ & & & $7(87.5)$ & & \\
\hline Bathing & & 0.21 & 0.640 & & 6.33 & 0.173 \\
\hline Home & $20(100.0)$ & & & $7(87.5)$ & & \\
\hline River & $0(0.0)$ & & & $0(0.0)$ & & \\
\hline Home and River & $0(0.0)$ & & & $1(12.5)$ & & \\
\hline Hand wash style after toilet & & 1.13 & 0.286 & & 14.08 & 0.495 \\
\hline With water & 10(50.0) & & & $2(25.0)$ & & \\
\hline With water \&soap & $10(50.0)$ & & & $6(75.0)$ & & \\
\hline Water source for drink & & 0.00 & 1.000 & & 0.00 & 1.000 \\
\hline Tap & 20(100.0) & & & $8(100.0)$ & & \\
\hline River & $0(0.0)$ & & & $0(0.0)$ & & \\
\hline Tap and River & $0(0.0)$ & & & $0(0.0)$ & & \\
\hline Washing cloth & & 0.91 & 0.740 & & 0.00 & 1.000 \\
\hline Home & 20(100.0) & & & $8(100.0)$ & & \\
\hline River & $0(0.0)$ & & & $0(0.0)$ & & \\
\hline Home \&River & $0(0.0)$ & & & $0(0.0)$ & & \\
\hline
\end{tabular}

"_" = Not Done

and in Tanzania [15]. The difference in the study area, where the present study was conducted in urban setting might be a reason. The co-infection rate in this study was higher than a study done at China [9], which might be due to the start of anti-TB chemotherapy before stool examination in Chinese study and the difference in the study setting.

Intestinal parasites and $\mathrm{TB}$, either way, might be a risk factor one to the other [3]. TB patients harbour more intestinal parasites (protozoa and helminths) infections than $\mathrm{TB}$ free household contacts $(\mathrm{COR}=2.85 ; 95 \% \mathrm{CI}=$ 1.18-6.87) in this study, suggesting that individuals with parasite infections are more susceptible to get TB. Similarly this was reported by previous studies conducted in different settings $[3,11,14]$. However; there was no difference between persons with PTB and healthy controls in a study from rural China [10], which might be due to the start of anti-TB chemotherapy before stool examination. In our study, the rate of intestinal helminths is significantly higher among $\mathrm{TB}$ patients, $(\mathrm{COR}=5.37$;
95\% CI $=1.14-25.25)$. The studies by Elias et al., in Gondar, Ethiopia [16] and Tristão- Sá et al., in Brazil [14] support our finding. However, the study conducted in Gondar by Abate et al., in Gondar, Ethiopia [7] and in Tanzania by Mhimbira et al., in Tanzania [15] did not show statisticially significant difference between the TB patients and TB free household contacts. This might be due to the variability in the epidemiology of intestinal helminths in various geographic locations. Moreover, in the present study we used highly sensitive diagnostics; Xpert MTB/RIF Assay and MGIT 960 liquid culture, to rule out TB from the study controls. Multiple parasitic infection in TB patients was about 4 $\%$ in the present study. Similarly, mixed or more than one species of intestinal parasites co-infection among TB patients were reported by Alemu et al., in Arbaminch, Ethiopia [12], Alemayehu et al., in Gondar, Ethiopia [11] and by Mhimbira et al., in Tanzania [15].

In this study; G.lamblia, A. lumbricoides, and H.ana were found with a significant difference in $\mathrm{TB}$ patients 
compared with controls. Likewise; a greater frequency of G.lambia infection in TB patients was reported by a study done at Gambo which is $250 \mathrm{~km}$ south of Addis Ababa, Ethiopia [30]. Similarly, A.lumbricoides was reported as the predominantly identified helminths in previous studies done in Ethiopia at Gondar [1, 3, 7] and Arbaminch [12]. Even though H.nana was significantly associated with TB in the present study, supportive findings are not found, which might be due to the absence of previous studies in similar settings. However, all the intestinal parasites identified in the present study except C.parvum was reported by different studies done in different settings and population $[3,5,7,10-13,15]$. The detection of C.parvum in TB patients in the present study might be due to the use of Modified Ziehl Neelsen staining method which was not included in the previous studies.

In the present study, intestinal parasitic infection on TB patients had a statistically significant association with BMI (AOR $=6.71 ; 95 \% \mathrm{CI}=1.65-27.25)$, which is supported by previous studies $[10,12,15]$. Even though, all two PTB patients from rural setting in this study were infected with intestinal parasites, it is difficult to compare with urban participants due to bigger numerical differences. However, studies from different parts of Ethiopia showed people who were living in rural areas were at risk of harboring intestinal parasites compared to urban dwellers $[11,12,31]$. Those who had dirty material in their finger nails were about nine times as likely to have intestinal parasites infection compared to those who did not have ( $\mathrm{AOR}=8.99 ; 95 \% \mathrm{CI}=2.46-32.78)$. This was supported by a study done by Abera et al., in Tilili, Ethiopia [32]; where $47.9 \%$ of students who had dirty material in their finger nails were infected with helminthes infection. In the control group, there was no variable significantly associated with intestinal parasitic infection. Similarly; in a study done in China [10], annual labor time in farmlands more than 2 months was the only risk factor associated with overall infections in healthy controls. The limitations of this study were; only one-time stool specimen examined, Katho Katz technique not used to assess the parasitic load, nutritional assessments except BMI were not done and HIV antibody testing was not done for controls. Even though it is valid to assess BCG vaccination status by looking scars, it is sometimes hard to see. All these factors might have a potential bias and un assessed confounding.

\section{Conclusion}

Although the study has limitations potentially leading to underdiagnosis due to one stool specimen is taken, the infection rate of intestinal parasites in TB patients and healthy household contacts had statistically significant difference. The most frequently identified intestinal parasites in TB patients with a statistical significant difference compared to controls were G.lamblia, A. lumbricoides and H.nana. Multiple parasitic infections were observed in TB patients but not in controls. Intestinal parasitic co-infection on TB patients had significant association with BMI and presence of dirty material in the participants's finger nail. The consequence of co-infection on developing an active disease, disease severity and treatment efficacy needs to be investigated in future. A large-scale study with diverse population and wide geographical coverage should done.

\section{Abbreviations \\ AIDS: Acquired Immunodeficiency Syndrome; AOR: Adjusted Odds Ratio; BCG: Bacilli Calmete Gurine; BMI: Body Mass Index; Cl: Confidence Interval; COR: Crude Odds Ratio; DOTs: Direct Observed Treatment short course; HIV: Human Immunodeficiency Virus; IP: Intestinal Parasites; LJ: Lowenstein Jenson; MGIT: Mycobacterium Growth Indicator Tube; MTB: Mycobacterium tuberculosis; NALC: N-Acetyl L-Cysteine; NaOH: Sodium Hydroxide; PTB: Pulmonary Tuberculosis; SPSS: Statistical Package for Social Sciences; TB: Tuberculosis; Th1: T helper type 1; WHO: World Health Organization; ZN: Ziehl Neelsen}

\section{Acknowledgements}

We would like to acknowledge: Department of Medical Laboratory Sciences, College of Health Sciences, Addis Ababa University, Ethiopian Public Health Institute, data collectors and study participants.

\section{Funding}

All the laboratory reagents, supplies and equipments are gained from Ethiopian Public Health Institute. Costs for design of study, data collection, analysis and interpretation of data are covered by Ethiopian Public Health Institute and Addis Ababa University.

Availability of data and materials

All original raw data is available in the corresponding author.

\section{Authors' contributions}

AA designed the study, analyzed results and wrote the manuscript. All works of this study was through the pertinent advice of $A K$ and $K D . B D, M A, G D$, $B Y, E T, M T, W S$ and DC participated in the data collection and laboratory examinations. All authors read and approved the final manuscript.

\section{Ethics approval and consent to participate}

The study obtained ethical approval from Department Research and Ethical Review Committee of the Department of Medical Laboratory Science, College of Health Sciences, Addis Ababa University. Permission letter was obtained from administrators of each facility. Participants were recruited after they became informed about the objectivesof the study and after they gave informed consent. For participants less than 18 years of age, their parents signed a written parental permission form. All participants who were positive for TB were linked with DOTs with free of charge and participants positive for intestinal paraites were treated accordingly. Since all study collection sites have routine anti-retroviral therapy ART) services, all HIV positive study participants were linked to each ART clinic through TB clinic focal persons for ART treatment initiation and management.

Consent for publication

Not applicable

Competing interests

The authors declared that, they have no any competing interests in the publication of this study.

\section{Publisher's Note}

Springer Nature remains neutral with regard to jurisdictional claims in published maps and institutional affiliations. 
Received: 20 November 2018 Accepted: 22 April 2019

Published online: 10 May 2019

\section{References}

1. WHO. Global Tuberculosis Report. Geneva: World Health Organization; 2017.

2. WHO. Global Tuberculosis Report. Geneva: World Health Organization; 2013.

3. Elias D, Mengistu G, Akuffo H, Britton S. Are intestinal helminths risk factors for developing active tuberculosis. Trop Med Int Health. 2006;11(4):551-8.

4. Brighenti S and Lerm M. How Mycobacterium tuberculosis Manipulates Innate and Adaptive Immunity - New Views of an Old Topic, Understanding Tuberculosis - Analyzing the Origin of Mycobacterium tuberculosis Pathogenicity. InTech. 2012; ISBN: 978-953-307-942-4. 207-34.

5. Neto MS, Totino RP, Sant'Anna MF, Coelho OV, Rolla CV, Zanini MG, et al. Enteroparasitosis prevalence and parasitism influence in clinical outcomes of tuberculosis Patientswith or without HIV co-infection in a reference Hospital in Rio de Janeiro (2000-2006). Braz J Infect Dis. 2009;13(6):427-32.

6. Kamal MS, Khalifa EK. Immune modulation by helminthic infections: worms and viral infections. Parasite Immunol. 2006;28(10):483-96.

7. Abate E, Belayneh M, Gelaw A, Idh J, Getachew A, Alemu S, et al. The impact of asymptomatic helminth co-infection in patients with newly diagnosed tuberculosis in north- West Ethiopia. PLoS One. 2012;7(8):e42901. https://doi.org/10.1371/journal.pone.0042901.

8. Hailu T. Current prevalence of intestinal parasites emphasis on hookworm and Schistosoma mansoni infections among patients at Workemeda health center, Northwest Ethiopia. Clin Microbial. 2014;3:4.

9. Gebreegziabiher D, Desta K, Howe R \& Abebe M. Helminth Infection Increases the Probability of Indeterminate QuantiFERON Gold in Tube Results in Pregnant Women. Bio Med Research International. 2014; 2014 Article ID 364137, 5 pages.

10. Li XX, Chen JX, Wang LX, Liu J, Wang FF, Wang Y, et al. Intestinal parasite co-infection among pulmonary tuberculosis cases without human immunodeficiency virus infection in a Rural County in China. Am J Trop Med Hyg. 2014;90(1):106-13.

11. Alemayehu M, Birhan W, Belyhun Y, Sahle M, Tessema B. Prevalence of smear positive tuberculosis, intestinal parasites and their co-infection among tuberculosis suspects in Gondar University hospital and Gondar poly clinic, north West Ethiopia. J Microb Biochem Technol. 2014;6(4):179-84.

12. Alemu G, Mama M. Intestinal helminth co-infection andassociated factors among tuberculosis patients in Arba Minch, Ethiopia. BMC Infect Dis. 2017;17:68.

13. Kassu A, Mohammad A, Fujimaki Y, Moges F, Elias D, Mekonnen F, et al. Serum IgE levels of tuberculosis patients in a tropical setup with high prevalence of HIV and intestinal parasitoses. Clin Exp Immunol. 2004;138: 122-7.

14. Tristão-Sá R, Rodrigues RR, Johnson LT, Pereira FL, Dietze R. Intestinal nematodes and pulmonary tuberculosis. Rev Soc Bras Med Trop. 2002;35: 533-5.

15. Mhimbira F, Hella J, Said K, Kamwela L, Sasamalo M, Maroa T, et al. Prevalence and clinical relevance of helminth co-infections among tuberculosis patients in urban Tanzania. PLoS Negl Trop Dis. 2017;11(2): e0005342. https://doi.org/10.1371/journal.pntd.0005342.

16. Li XX, Zhou XN. Co-infection of tuberculosis and parasitic diseases in humans: a systematic review. Parasit Vectors. 2013;6:79.

17. Nutman TB, Kumaran P, Chandrasekaran V, George JP, Anuradha R. Modulation of mycobacterial-specific Th1 and Th17 cells in latent tuberculosis by coincident hookworm infection. J Immunol. 2013;190:5161-8.

18. Babu S, Bhat SQ, Kumar PN, Jayantasri S, Rukmani S, Kumaran P, et al. Human type 1 and 17 responses in latent tuberculosis are modulated by coincident filarial infection through cytotoxic T lymphocyte antigen-4 and programmed death-1. J Infect Dis. 2009;200:288-98.

19. City Government of Addis Ababa Bureau of Finance and Economic Development. Socio-Economic Profile of Addis Ababa for the Year 2004 E.C/ 2011/12G.C. AA BoFED.2013. Available at http://www.addisababa.gov.et/fi/ web/guest/home. Accessed 5 May 2016.

20. Federal Democratic Republic of Ethiopia Ministry of Health; National Comprehensive Tuberculosis, Leprosy and TB/HIV Training Manual for Health Care Workers. 2016. Accessed from: https://www.slideshare.net/ suleymanfantahun/new-ethiopian-tb-guildline-november-2016. Accessed 20 Dec 2016
21. Goyal R\& Kumar A. A comparison of Ziehl-Neelsen staining and fluorescent microscopy for diagnosis of pulmonary tuberculosis. IOSR J Dent Med Sci 2013; 8(5): 05-08.

22. GLI. Mycobacteriology laboratory manual. Global Laboratory Initiative. 2014:1-154.

23. Esmael A, Ali I, Agonafi M, Endris M, Getahun M, Desta K, et al. Drug resistance pattern of Mycobacterium tuberculosis in eastern Amhara regional state, Ethiopia. J Microb Biochem Technol. 2014;6(2):075-9.

24. Kanade S, Nataraj G, Suryawanshi R, Mehta P. Utility of MPT 64 antigen detection assay for rapid characterization of mycobacteria in a resource constrained setting. Indian J. Tuberc. 2012;59(2):92-6.

25. Cephied: Xpert ${ }^{\oplus}$ MTB/RIF, two-hour detection of MTB and resistance to rifampicin. In Vitro Diagnostic Medical Device. 2009; 300-7810 Rev. A:1-16.

26. Cheesbrough M. District laboratory practice in tropical countries, part 1, second edition. Cambridge: Cambridge university press; 2009. ISBN-13 9780511349355

27. Casemore DP. Laboratory methods for diagnosing Cryptosporidioosis. J Clin Pathol. 1991;44:445-51.

28. Federal Ministry of Health of Ethiopia:Implementation Guideline for TB/HIV Collaborative Activities in Ethiopia. 2007. Accessed from: https://www.who. int/hiv/pub/guidelines/ethiopia.pdf. Accessed 12 June 2016.

29. Siddiqi SH\&Gerdes SH. MGITM procedure manual. Geneva: Foundation for Innovative New Diagnostics (FIND); 2006.

30. Ramos JM, Reyes F, Tesfamariam A. Intestinal parasites in adults admitted to a rural Ethiopian hospital: relationship to tuberculosis and malaria. Scand J Infect Dis. 2006;38:460-2.

31. Alemu M, Kinfe B, Tadesse D, Mulu W, Hailu T, Yizengaw E. Intestinal parasitosis and anaemia among patients in a health center, North Ethiopia. BMC Res Notes. 2017;10:632.

32. Abera A, Nibret E. Prevalence of gastrointestinal helminthic infections and associated risk factors among school children in Tilili town, Northwest Ethiopia. Asian Pac J Trop Med. 2014:525-30.

Ready to submit your research? Choose BMC and benefit from:

- fast, convenient online submission

- thorough peer review by experienced researchers in your field

- rapid publication on acceptance

- support for research data, including large and complex data types

- gold Open Access which fosters wider collaboration and increased citations

- maximum visibility for your research: over $100 \mathrm{M}$ website views per year

At BMC, research is always in progress.

Learn more biomedcentral.com/submissions 